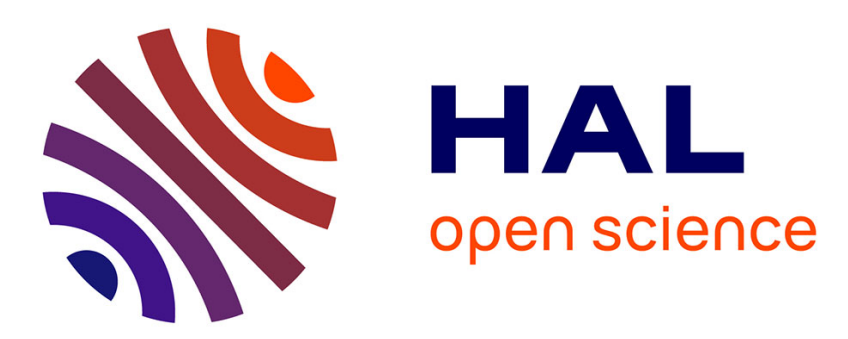

\title{
Une méthode de marquage individuel des coléoptères scolytides pour les études de laboratoire
}

\author{
François Lieutier, Raymond Ham, Marie-Claude Ham, Jacques Garcia
}

\section{To cite this version:}

François Lieutier, Raymond Ham, Marie-Claude Ham, Jacques Garcia. Une méthode de marquage individuel des coléoptères scolytides pour les études de laboratoire. Agronomie, 1986, 6 (8), pp.773776. hal-00884936

\section{HAL Id: hal-00884936 https://hal.science/hal-00884936}

Submitted on 1 Jan 1986

HAL is a multi-disciplinary open access archive for the deposit and dissemination of scientific research documents, whether they are published or not. The documents may come from teaching and research institutions in France or abroad, or from public or private research centers.
L'archive ouverte pluridisciplinaire HAL, est destinée au dépôt et à la diffusion de documents scientifiques de niveau recherche, publiés ou non, émanant des établissements d'enseignement et de recherche français ou étrangers, des laboratoires publics ou privés. 


\title{
Une méthode de marquage individuel des coléop- tères scolytides pour les études de laboratoire
}

François LIEUTIER, Raymond HAM, Marie-Claude HAM \& Jacques GARCIA

I.N.R.A., Station de Zoologie forestière, Centre de Recherches d'Orléans, Ardon, F 45160 Olivet

RÉSUMÉ

\begin{abstract}
Une technique de marquage individuel par gravage de la face dorsale des élytres des Scolytides est proposée. Elle permet de différencier individuellement un millier d'insectes. Elle provoque une légère mortalité, mais l'envol et le comportement de ponte des insectes survivants n'est pas modifié. Elle paraît bien adaptée à des études de laboratoire ou à des études de faibles dimensions sur le terrain.
\end{abstract}

Mots clés additionnels : Marquage, Scolytidae, ponte, vol, survie.

Individual tagging of bark beetles for laboratory studies.

A method for tagging bark beetles by engraving the elytra is proposed. One thousand beetles could be individually marked. Slight mortality was observed, but the flight and the oviposition of surviving beetles was not affected. The method seems convenient for laboratory studies and short-range field studies.

Additional key words : Tagging, Scolytidae, oviposition, flight, survival.

\section{INTRODUCTION}

Lors d'études sur les populations de coléoptères menées en laboratoire ou en cage sur le terrain, il s'avère fréquemment indispensable de pouvoir déterminer avec une certaine précision l'âge des insectes (séparation des parents de leurs descendants par exemple). De même, il est souvent utile de pouvoir suivre individuellement le devenir de chaque insecte, lors de ses déplacements ou pour des études de longévité par exemple. Une technique fine de marquage constitue donc un outil précieux pour mener à bien ces études. Dans le cas des scolytides, diverses méthodes de marquage ont été expérimentées relativement récemment, tant sur le terrain qu'au laboratoire: marquage radioactif par le phosphore 32 pour l'étude de la dispersion de divers Dendroctonus par exemple (SCHMID, 1970 ; MOORE \& TAYLOR, 1976 ; MOORE et al., 1979) ; marquage à la peinture ou au vernis (HERTEL et al., 1969; WITANACHCHI, 1980). Toutefois, ces méthodes ne permettent pas de différencier les insectes individuellement. Aussi proposons nous ici une méthode fondée sur le gravage de la cuticule des scolytes. Ceux-ci possèdent le plus souvent des rangées longitudinales de ponctuations élytrales qui permettent un repérage aisé dans le cas d'une numérotation individuelle.

\section{MATÉRIEL ET MÉTHODES}

Les tests ont porté sur Ips sexdentatus Boern.

\section{A. Marquage}

Deux techniques sont comparées : vernis et gravage. Deux gouttes de vernis à ongle coloré sont appliquées sur les élytres de chaque scolyte, l'une dorsalement, l'autre dans la déclivité. Pour le gravage, sur chaque insecte, une petite entaille linéaire est exécutée à la lame de rasoir sous la loupe binoculaire, entre 2 lignes de ponctuation sur la face dorsale des élytres.

\section{B. Persistance des marques et survie des insectes}

Des insectes hivernants sont récoltés sur le terrain mi-novembre et mis en éclosoir entre écorces de pin sylvestre jusqu'à ce qu'ils émergent. Ils sont alors soit marqués (vernis ou gravage), soit laissés sans marques. Chaque catégorie est ensuite installée séparément sur des rondins frais de pin sylvestre. Après pénétration des insectes, une partie des rondins est remise dans la nature sous sac de toile jusqu'en avril, l'autre est laissée au laboratoire jusqu'en mars. Dans ce dernier cas, afin que les insectes disposent d'une 
quantité suffisante de nourriture, des rondins frais sont ajoutés chaque mois dans les enceintes d'élevage. A la fin de l'expérimentation, les résultats sont lus par écorçage total de tous les rondins et inventaire des insectes récupérés.

\section{Action du marquage sur l'émergence, l'envol et la ponte}

Pour chacune des 2 générations annuelles, des insectes sont récoltés dans la nature peu de temps avant leur émergence, une partie est marquée par gravage. Les insectes témoins subissent les mêmes manipulations sous la loupe que les insectes marqués. Les morceaux d'écorce ayant contenu les insectes sont portés brutalement à $-35^{\circ} \mathrm{C}$ pendant une nuit de façon à tuer les Ips qui n'auraient pas été récupérés. Après retour des écorces à température normale, les insectes y sont réintroduits et l'ensemble est placé en éclosoir, en 2 lots à $22-25^{\circ} \mathrm{C}$, insectes marqués d'une part, non marqués d'autre part, pour que tous y terminent leur maturation, jusqu'à émergence. L'aptitude à l'envol est appréciée sur les émergents par leur capacité à s'envoler à $22-25^{\circ} \mathrm{C}$ face à une vitre éclairée en lumière naturelle. Dans chacune des catégories, les insectes ayant montré une aptitude à l'envol sont introduits sur rondins de pin sylvestre frais pour juger de leur capacité reproductrice. Pour cela, les mâles sont placés chacun dans un avant-trou foré mécaniquement à travers l'écorce ; une femelle est introduite avec chaque mâle $24 \mathrm{~h}$ plus tard. L'ensemble est laissé en évolution à $22-25^{\circ} \mathrm{C}$ au laboratoire pendant 2 semaines. Ce laps de temps écoulé, les rondins sont écorcés et les caractéristiques des pontes notées : présence d'une ponte, longueur de la galerie maternelle depuis la chambre d'accouplement jusqu'à la $1^{\text {re }}$ encoche de ponte, nombre total d'encoches de ponte, densité de ponte (nombre d'encoches par $\mathrm{cm}$ de galerie de ponte).

\section{RÉSULTATS}

\section{A. Evolution des marques}

Dans le cas d'un marquage au vernis, la surface marquée est toujours beaucoup plus faible après récupération des insectes que lors du marquage. Dans cer- tains cas, elle ne persiste que sur le dos ou dans la déclivité, quand elle ne disparaît pas complètement (cf. infra). Lors du marquage par gravage, il n'est pas rare que l'entaille dans l'élytre provoque un léger écoulement d'hémolymphe, surtout chez les individus dont la mélanisation n'est pas terminée. Les marques évoluent ensuite en brunissant puis noircissant, la mélanisation correspondant à une réaction de cicatrisation.

\section{B. Persistance des marques et survie des insectes}

Les tableaux 1 et 2 rendent compte des résultats obtenus respectivement sur le terràin et au laboratoire. Une proportion plus faible d'insectes marqués que non marqués survit à l'hiver sur le terrain, la différence étant significative dans le cas du marquage par gravage. Les marques sont retrouvées chez tous les insectes gravés mais non chez tous ceux qui portaient du vernis, la différence entre ces 2 catégories étant hautement significative. Au laboratoire, un plus grand nombre d'insectes gravés que d'insectes vernis est retrouvé à la fin de l'expérience, 3 d'entre eux étant toujours vivants. En outre, l'écorçage des rondins introduits en mi-novembre et mi-décembre permet de constater qu'ils ne renferment plus qu'un faible nombre d'insectes en mars, mais qu'ils ont permis auparavant la multiplication et le développement d'un grand nombre d'individus. Les rondins introduits en janvier et février renferment en revanche, en mars, une population importante tant au stade œuf, larvaire ou nymphal, qu'adulte.

\section{L'émergence et l'envol (tabl. 3)}

Pour chacune des 2 générations, les insectes marqués émergent en plus faible pourcentage que les nonmarqués, la différence étant significative pour l'émergence d'été. Les pourcentages d'émergence sont par ailleurs plus élevés en été qu'au printemps, dans le cas des insectes non marqués. Aucune différence significative n'existe en revanche entre les insectes marqués et les témoins, quant à leur aptitude à l'envol.

\section{Les caractéristiques de la ponte (tabl. 4)}

Pour aucune des caractéristiques relevées, il n'a pu être observé de différence entre les insectes marqués et

TABLEAU

Survie des insectes et persistance des marques après 4 mois $1 / 2$ d'hiver sur le terrain. Les intervalles de confiance sont calculés pour un coefficient de sécurité de $95 \mathrm{p}$. 100. Les valeurs affectées de (a) diffèrent entre elles significativement au seuil de 95 p. 100 ; celles affectées de (b) diffèrent significativement au seuil de 99 p. 100.

Bark beetle survival and persistence of marks after 4.5 months in winter in the field. $95 \%$ confidence intervals; (a) significant difference at the $95 \%$ level, (b) at $99 \%$ level.

\begin{tabular}{lccc}
\hline \hline & Non marqués & Marqués & par gravage \\
\hline Nombre d'insectes introduits fị novembre & 47 (sex-ratio 1,75) & 46 (sex-ratio 1,89) & 46 (sex-ratio 1,56) \\
\hline Nombre d'insectes retrouvés en avril (vivants ou morts) & 43 & 41 & 35 \\
\hline $\begin{array}{l}\text { \% de survie insectes vivants/insectes introduits } \\
\text { Persistance du marquage (\% d'insectes avec marque, } \\
\text { vivants ou morts) }\end{array}$ & $\begin{array}{c}61,7 \pm 14,2(\mathrm{a}) \\
\text { (sex-ratio 1,64) }\end{array}$ & $\begin{array}{c}54,3 \pm 14,7 \\
(\text { sex-ratio } 1,50)\end{array}$ & $\begin{array}{c}39,1 \pm 114,4(\mathrm{a}) \\
(\text { sex-ratio } 1,57)\end{array}$ \\
\hline \hline
\end{tabular}


TABLEAU 2

Persistance du marquage et survie des insectes au laboratoire après 4 mois d'activité intense.

Persistence of marks and bark beetle survival in the laboratory after 4 months of intense activity.

\begin{tabular}{|c|c|c|c|c|c|c|c|c|c|c|}
\hline \multirow{3}{*}{$\begin{array}{c}\text { Nombre d'insectes } \\
\text { introduits en novembre } \\
\begin{array}{c}\text { Nombre d'insectes adultes } \\
\text { retrouvés en mars }\end{array}\end{array}$} & \multicolumn{2}{|c|}{$\begin{array}{l}\text { Non marqués } \\
12 \text { ơ } 30 \%\end{array}$} & \multicolumn{8}{|c|}{$\begin{array}{l}\text { par gravage } \\
110^{*} 30 \%\end{array}$} \\
\hline & \multirow[b]{2}{*}{ vivants } & \multirow[b]{2}{*}{ morts } & \multicolumn{2}{|c|}{ marqués } & \multicolumn{2}{|c|}{ non marqués } & \multicolumn{2}{|c|}{ marqués } & \multicolumn{2}{|c|}{ non marqués } \\
\hline & & & vivants & morts & vivants & morts & vivants & morts & vivants & morts \\
\hline $\begin{array}{l}\text { dans la cage d'élevage } \\
\text { en dehors des rondins }\end{array}$ & 0 & 16 & 0 & 1 & 1 & 17 & 0 & 1 & 2 & 9 \\
\hline $\begin{array}{l}\text { dans rondins introduits } \\
\text { en novembre et décembre }\end{array}$ & 7 & 16 & 0 & 4 & 7 & 18 & 0 & 12 & 21 & 10 \\
\hline $\begin{array}{l}\text { dans rondins introduits } \\
\text { en janvier et février }\end{array}$ & 486 & 22 & 0 & 0 & 247 & 5 & 3 & 3 & 534 & 26 \\
\hline total marqués & & & 0 & 5 & & & 3 & 15 & & \\
\hline
\end{tabular}

TABLEAU 3

Emergence et capacité d'envol des insectes marqués par gravage et des insectes témoins. Les intervalles de confiance sont calculés pour un coefficient de sécurité de $95 \mathrm{p}$. 100. Les pourcentages affectés de la même lettre diffèrent entre eux de façon significative au seuil de $99 \mathrm{p}$. 100. Les nombres d'insectes testés sont indiqués entre parenthèses.

Emergence and flying ability of the engraved and control beetles. $95 \%$ confidence intervals.

Percentages with the same letter differ significantly at the $99 \%$ level. Numbers of beetles in brackets.

\begin{tabular}{lcccc}
\hline \hline & \multicolumn{2}{c}{ Essaimage de printemps } & \multicolumn{2}{c}{ Essaimage d'été } \\
& non marqués & marqués & non marqués & marqués \\
\hline$\%$ d'émergence & $76,3 \pm 6,8(\mathrm{~b})$ & $73,2 \pm 6,4$ & $95,2 \pm 3,3(\mathrm{ab})$ & $80,7 \pm 6,4(\mathrm{a})$ \\
& $(173)$ & $(190)$ & $(168)$ & $(150)$ \\
& $90,4 \pm 6,9$ & $90,1 \pm 6,6$ & $83,3 \pm 6,3$ & $91,3 \pm 5,9$ \\
$(73)$ & $(81)$ & $(138)$ & $(92)$ \\
\hline
\end{tabular}

TABLEAU 4

Caractéristiques de la ponte des insectes marqués par gravage et des insectes témoins. Les nombres d'insectes testés sont indiqués entre parenthèses. Les intervalles de confiance sont calculés pour un coefficient de sécurité de $95 \mathrm{p} .100$. Aucune différence entre insectes marqués et non marqués ou entre printemps et été n'est significative.

Oviposition characteristics of the engraved and control beetles. Numbers of insects in brackets. $95 \%$ confidence intervals. Differences between engraved and control beetles or between spring and summer generations were never significant.

\begin{tabular}{|c|c|c|c|c|}
\hline & \multicolumn{2}{|c|}{ Printemps } & \multicolumn{2}{|c|}{ Eté } \\
\hline & non marqués & marqués & non marqués & marqués \\
\hline$\%$ femelles ayant pondu & $100(28)$ & $100(31)$ & $100(26)$ & $100(20)$ \\
\hline $\begin{array}{l}\text { Longueur de la galerie maternelle } \\
\text { jusqu'à la } 1^{\text {re }} \text { encoche }(\mathrm{mm})\end{array}$ & $\begin{array}{l}14,3 \pm 3,0 \\
(28)\end{array}$ & $\frac{13,6 \pm 3,0}{(22)}$ & $\underset{(26)}{11,2 \pm 1,7}$ & $\begin{array}{c}11,8 \pm 3,1 \\
(20)\end{array}$ \\
\hline Nombre d'encoches de ponte & $\begin{array}{l}30,4 \pm 5,8 \\
(28)\end{array}$ & $32,3 \pm 5,6$ & $\begin{array}{l}33,3 \pm 4,7 \\
(26)\end{array}$ & $\begin{array}{l}40,0 \pm 8,2 \\
(20)\end{array}$ \\
\hline $\begin{array}{l}\text { Densité des encoches de ponte } \\
\text { (nombre/cm galerie) }\end{array}$ & $\begin{array}{l}2,2 \pm 0,2 \\
\quad(28)\end{array}$ & $\begin{array}{c}2,5 \pm 0,35 \\
(22)\end{array}$ & $\begin{array}{l}2,5 \pm 0,3 \\
(26)\end{array}$ & $\begin{array}{c}2,5 \pm 0,4 \\
(20)\end{array}$ \\
\hline
\end{tabular}

témoins. Les valeurs obtenues sont même le plus souvent remarquablement semblables. On note généralement des différences entre générations d'été et de printemps (plus grand nombre d'œufs et plus faible longueur de la galerie avant la $1^{\text {re }}$ encoche pour la génération d'été) mais elles ne sont pas significatives.

\section{DISCUSSION ET CONCLUSION}

Il est indéniable que la technique de gravage cause une certaine mortalité. C'est ce qui ressort des résul- tats des tableaux 1 et 3 . Les 2 sexes sont affectés. Cette mortalité est due probablement, au moins en partie, au stress causé par les manipulations des insectes sous la loupe binoculaire, ce qui expliquerait la moins bonne survie des insectes vernis par rapport aux témoins, bien que la différence ne soit pas significative. Cependant, la différence relativement importante entre les insectes marqués au vernis et ceux marqués par gravage met en cause le gravage lui-même. La légère blessure infligée par cette technique est sans doute à l'origine de cette mort, probablement plus par 
suite d'infections contractées par cette voie que par suite de la perte d'hémolymphe elle-même. Toutefois, quand le stress climatique ou comportemental n'accompagne pas la blessure, la mortalité, bien que significative, est relativement limitée (tabl. 3). La différence significative observée dans le tableau 3 entre le pourcentage d'émergence des insectes témoins de la génération d'été et ceux de la génération d'hiver, souligne d'ailleurs l'importance du stress climatique subi par les insectes quand on les conduit du terrain au laboratoire. Après cicatrisation, les insectes gravés survivants montrent en revanche un comportement d'envol et de ponte absolument semblable à celui des témoins.

La persistance des marques gravées est excellente, tant en conditions d'hivernation (tabl. 1) qu'en conditions d'activité intense au laboratoire (tabl. 2). Dans ce dernier cas en effet, le temps écoulé depuis l'installation jusqu'à l'écorçage et l'existence en mars d'un grand nombre de descendants adultes sur les rondins introduits en janvier et février tandis que ceux de novembre et décembre sont abandonnés, laissent penser que 2 générations successives ont eu lieu, la $1^{\text {re }}$ sur les rondins de novembre et décembre, la $2^{\mathrm{e}}$ sur ceux de janvier et février. On sait en effet que le développement complet d'une génération prend environ 1,5 à 2 mois dans les conditions du laboratoire. Après un tel délai et une telle activité, la plupart dęs insectes introduits en novembre sont morts et le fait que l'on ne retrouve, dans le tableau 2, que 18 insectes marqués sur 41 introduits s'explique précisément par cette activité sous-corticale intense, accompagnée d'une destruction et d'une décomposition rapide des insectes morts.

Ainsi, bien qu'il existe une certaine mortalité, la persistance des marques et l'identité de comportement des insectes marqués et des insectes témoins permettent de considérer que la méthode du gravage est utilisable avec profit. Le rendement peut même être très bon si l'on prend la précaution de ne pas faire subir de stress climatique aux insectes. Il convient cependant de ne marquer que des insectes suffisamment mélanisés pour éviter une blessure trop importante. En outre, en positionnant les marques de manière variable le long des élytres (par exemple partie antérieure, moyenne ou postérieure) et en utilisant les rangées de ponctuations comme repère d'un système de numérotation, on peut procéder à un marquage individuel des insectes. Dans le cas d'Ips sexdentatus, qui possède un grand nombre de rangées de ponctuations, il est ainsi aisé de délimiter 10 zones, comprises entre ces rangées, dans lesquelles le gravage est possible. On peut alors individualiser très facilement un millier d'insectes différents dans un système à base 10 , en n'effectuant pas plus de 3 stries par insecte.

Le marquage par gravage nous paraît donc une méthode parfaitement adaptée aux besoins des études en laboratoire ou en cage d'élevage dans la nature. Malheureusement, ses possibilités d'utilisation à grande échelle sur le terrain, pour des études de dispersion par exemple, paraissent limitées. En effet, bien que le procédé soit rapide en lui-même (une personne expérimentée peut marquer individuellement une centaine d'insectes en $1 \mathrm{~h}$ ), la nécessité de marquer les milliers d'insectes indispensables aux lâchers en forêt rend l'opération assez lourde pour de tels projets et impose le stockage des insectes au laboratoire pendant un certain temps, ce qui risque de compromettre leur survie.

Reçu le 28 janvier 1986. Accepté le 28 avril 1986.

\section{RÉFÉRENCES BIBLIOGRAPHIQUES}

Hertel G. D., Hain F. P., Anderson R. F., 1969. Response of Ips grandicollis (Coleoptera : Scolytidae) to the attractant produced by attacking male beetles. Can. Entomol., 101, 1084-1091.

Moore G. E., Taylor J. F., 1976. Tagging of the southern pine beetle with Phosphorus 32. Environ. Entomol., 5, 1065-1067.

Moore G. E., Taylor J. F., Smith J., 1979. Tracing dispersion of southern pine beetles from felled brood trees with Phosphorus 32 . J. Ga. entomol. Soc., 14, 83-87.
Schmid J. M., 1970. Dispersal studies with radioactively tagged spruce beetles. U. S. For. Serv. Res. Note $\mathrm{n}^{\circ}$ R. M. 178, 3 p.

Witanachchi J. P., 1980. Evidence for pre-emergence mating among mature progeny of Ips grandicollis (Eichhoff). J. Aust. entomol. Soc., 19, 93-100. 